\title{
Design New Intelligent-Base Chattering Free Nonlinear Control of Spherical Motor
}

\author{
Mohammad Ali Tayebi, Farzin Piltan, Mahsa Piltan, Mojtaba Yaghoot, Meysam Es maeili \\ Research and Development Department, Institute of Advance Science and Technology-IRAN SSP, Shiraz, Iran \\ http://www.iranssp.com, Email: ssp.robotic@iranssp.com
}

\begin{abstract}
The main four objectives to design controllers are: stability, robust, minimum error and reliability. Linear PID controller is model-free controller and this controller is not reliable. One of the robust nonlinear controller to control of nonlinear systems is sliding mode controller (SMC). Sliding mode controller (SMC) is robust conventional nonlinear controller in a partly uncertain dynamic system's parameters. Sliding mode controller is divided into two main sub parts: discontinues controller $\left(\boldsymbol{\tau}_{\text {dis }}\right)$ and equivalent controller $\left(\boldsymbol{\tau}_{\boldsymbol{e q}}\right)$. Discontinues controller is used to design suitable tracking performance based on very fast switching. Fast switching or discontinuous part have essential role to achieve to good trajectory following, but it is caused system instability and chattering phenomenon. Chattering phenomenon is one of the main challenges in conventional sliding mode controller and it can causes some important mechanical problems such as saturation and heats the mechanical parts of robot manipulators or drivers. To reduce or eliminate the chattering two methods are used in many researches which these methods are: boundary layer saturation method and artificial intelligence based method. In this research fuzzy switching methodology is used to eliminate the chattering in presence of uncertainty to increase the robust of this controller with application to three dimensions of spherical motor.
\end{abstract}

Index Terms- Fuzzy Sliding Mode Algorithm, Spherical Motor, Chattering Phenomenon, Fuzzy Logic Controller

\section{INTRODUCTION AND BACKGROUND}

Multi-degrees-of-freedom (DOF) actuators are wide used in a number of Industries. Currently, a significant number of the existing robotic actuators that can realize multi-DOF motion are constructed using gear and linkages to connect several single-DOF motors in series and/or parallel. Not only do such actuators tend to be large in size and mass, but they also have a decreased positioning accuracy due to mechanical deformation, friction and backlash of the gears and linkages. A nu mber of these systems also exhibit singularities in their works paces, which makes it virtually impossible to obtain uniform, high-speed, and high-precision motion. For high precession trajectory planning and control, it is necessary to replace the actuator system made up of several singleDOF motors connected in series and/or parallel with a single multi-DOF actuator. The need for such systems has motivated years of research in the development of unusual, yet high performance actuators that have the potential to realize multi-DOF motion in a single joint. One such actuator is the spherical motor. Compared to conventional robotic manipulators that offer the same motion capabilities, the spherical motor possesses several advantages. Not only can the motor comb ine 3-DOF motion in a single joint, it has a large range of motion with no singularities in its workspace. The spherical motor is much simpler and more compact in design than most multiple single-axis robotic manipulators. The motor is also relatively easy to manufacture. The spherical motor have potential contributions to a wide range of applications such as coordinate measuring, object tracking, material handling, automated as sembling, weld ing, and laser cutting. All these applications require high precision motion and fast dynamic response, which the spherical motor is capable of delivering. Previous research efforts on the spherical motor have demonstrated most of these features. These, however, come with a number of challenges. The spherical motor exhibits coupled, nonlinear and very complex dynamics. The design and implementation of feedback controllers for the motor are complicated by these dynamics. The controller design is further complicated by the orientation-varying torque generated by the spherical motor. Some of these challenges have been the focus of previous and ongoing research [1-11].

In modern usage, the word of control has many meanings, this word is usually taken to mean regulate, direct or command. The word feedback plays a vital role in the advance engineering and science. The conceptual frame work in Feed-back theory has developed only since world war II. In the twentieth century, there was a rapid growth in the application of feedback controllers in process industries. According to Ogata, to do the first significant work in three-term or PID controllers which Nicholas Minorsky worked on it by automatic controllers in 1922. In 1934, Stefen Black was invention of the feedback amplifiers to develop the negative feedback amplifier[12-28]. Negative feedback invited communications engineer Harold Black in 1928 and it occurs when the output is subtracted from the input. Automatic control has played an important role in advance science and engineering and its extreme importance in many industrial applications, i.e., aerospace, mechanical engineering and joint control. The first significant work in automatic control was James Watt's centrifugal governor for the speed control in motor engine in eighteenth century[29-40]. There are several methods for controlling a spherical motor, which all of them follow two common goals, namely, hardware/software implementation and 
acceptable performance. However, the mechanical design of spherical motor is very important to select the best controller but in general two types schemes can be presented, namely, a joint space control schemes and an operation space control schemes [41-55]. Joint space and operational space control are closed loop controllers which they have been used to provide robustness and rejection of disturbance effect. The main target in joint space controller is to design a feedback controller which the actual motion $\left(\boldsymbol{q}_{\boldsymbol{a}}(\boldsymbol{t})\right)$ and desired motion $\left(\boldsymbol{q}_{\boldsymbol{d}}(\boldsymbol{t})\right)$ as closely as possible. This control problem is classified into two main groups. Firstly, transformation the desired motion $\boldsymbol{X}_{\boldsymbol{d}}(\boldsymbol{t})$ to joint variable $\boldsymbol{q}_{\boldsymbol{d}}(\boldsymbol{t})$ by inverse kinematics of spherical motor[56-60]. This control includes simple PD control, PID control, inverse dynamic control, Lyapunov-based control, and passivity based control. The main target in operational space controller is to design a feedback controller to allow the actual endeffector motion $\boldsymbol{X}_{\boldsymbol{a}}(\boldsymbol{t})$ to track the desired endeffector motion $\boldsymbol{X}_{\boldsymbol{d}}(\boldsymbol{t})$. This control methodology requires a greater algorithmic complexity and the inverse kinematics used in the feedback control loop. Direct measurement of operational space variables are very expensive that caused to limitation used of this controller in spherical motor[5355]. One of the simplest ways to analysis control of three DOF spherical motor are analyzed each joint separately such as SISO systems and design an independent joint controller for each joint. In this controller, inputs only depends on the velocity and displacement of the corresponding joint and the other parameters between joints such as coupling presented by disturbance input. Joint space controller has many advantages such as one type controllers design for all joints with the same formulation, low cost hardware, and simple structure. A nonlinear methodology is used for nonlinear uncertain systems (e.g., spherical motor) to have an acceptable performance. These controllers divided into six groups, namely, feedback linearization (computed-torque control), passivity-based control, sliding mode control (variable structure control), artificial intelligence control, lyapunovbased control and adaptive control[13-26]. Sliding mode controller (SMC) is a powerful nonlinear controller which has been analyzed by many researchers especially in recent years. This theory was first proposed in the early 1950 by Emelyanov and several co-workers and has been extensively developed since then with the invention of high speed control devices [12-18]. The main reason to opt for this controller is its acceptable control performance in wide range and solves two most important challenging topics in control which names, stability and robustness [24-55]. Sliding mode controller is divided into two main sub controllers: discontinues controller $\left(\boldsymbol{\tau}_{\text {dis }}\right)$ and equivalent controller $\left(\boldsymbol{\tau}_{\boldsymbol{e q}}\right)$. Discontinues controller causes an acceptable tracking performance at the expense of very fast switching. In the theory of infinity fast switching can provide a good tracking performance but it also can provide some problems (e.g., system instability and chattering phenomenon). After going toward the sliding surface by discontinues term, equivalent term help to the system dynamics match to the sliding surface[12-15].
However, this controller used in many applications but, pure sliding mode controller has following challenges: chattering phenomenon, and nonlinear equivalent dynamic formulation [20]. Chattering phenomenon can causes some problems such as saturation and heat the mechanical parts of spherical motor. To reduce or eliminate the chattering, various papers have been reported by many researchers which classified into two most important methods: boundary layer saturation method and estimated uncertainties method [58-60]. In boundary layer saturation method, the basic idea is the discontinuous method replacement by saturation (linear) method with small neighborhood of the switching surface. This replacement caused to increase the error performance against with the considerable chattering reduction. In recent years, artificial intelligence theory has been used in sliding mode control systems. Neural network, fuzzy logic and neurofuzzy are synergically combined with nonlinear classical controller and used in nonlinear, time variant and uncertain plant (e.g., spherical motor). Fuzzy logic controller (FLC) is one of the most important applications of fuzzy logic theory. This controller can be used to control nonlinear, uncertain, and noisy systems. This method is free of some model techniques as in modelbased controllers. As mentioned that fuzzy logic application is not only limited to the modelling of nonlinear systems [31-36] but also this method can help engineers to design a model-free controller. Control spherical motor using model-based controllers are based on manipulator dynamic model. These controllers often have many problems for modelling. Conventional controllers require accurate information of dynamic model of spherical motor, but most of time these models are MIMO, nonlinear and partly uncertain therefore calculate accurate dynamic model is complicated [32]. The main reasons to use fuzzy logic methodology are able to give approximate recommended solution for uncertain and also certain complicated systems to easy understanding and flexible. Fuzzy logic provides a method to design a model-free controller for nonlinear plant with a set of IFTHEN rules [32]. This paper contributes to the research effort of alternate methods for modeling the torque generated by the spherical motor used in the fuzzy sliding mode-type feedback controller design. The designed controller not only demonstrates the appealing features exhibited by the spherical motor, but also demonstrates some of the nice features of fuzzy sliding mode-type controllers as well. This paper is organized as follows; second part focuses on the modeling dynamic formulation based on Lagrange methodology, sliding mode controller to have a robust control, and design fuzzy logic compensator. Third part is focused on the methodology which can be used to reduce the error, increase the performance quality and increase the robustness and stability. Simulation result and discussion is illustrated in forth part which based on trajectory following and disturbance rejection. The last part focuses on the conclusion and compare between this method and the other ones. 


\section{THEORY}

\section{Dynamic and Kinematics Formulation of Spherical Motor}

Dynamic modeling of spherical motors is used to describe the behavior of spherical motor such as linear or nonlinear dynamic behavior, design of model based controller such as pure sliding mode controller which design this controller is based on nonlinear dynamic equations, and for simulation. The dynamic modeling describes the relationship between motion, velocity, and accelerations to force/torque or current/voltage and also it can be used to describe the particular dynamic effects (e.g., inertia, coriolios, centrifugal, and the other parameters) to behavior of system[1-10]. Spherical motor is nonlinear and uncertain dynamic parameters and it is 3 degrees of freedom (DOF) electrical motor.

The equation of a spherical motor governed by the following equation [1-10]:

$$
H(q)\left[\begin{array}{c}
\ddot{\alpha} \\
\ddot{\beta} \\
\ddot{\gamma}
\end{array}\right]+B(q)\left[\begin{array}{c}
\dot{\alpha} \dot{\beta} \\
\dot{\alpha} \dot{\gamma} \\
\dot{\beta} \dot{\gamma}
\end{array}\right]+C(q)\left[\begin{array}{c}
\dot{\alpha}^{2} \\
\dot{\beta}^{2} \\
\dot{\gamma}^{2}
\end{array}\right]=\left[\begin{array}{c}
\tau_{x} \\
\tau_{y} \\
\tau_{z}
\end{array}\right]
$$

Where $\tau$ is actuation torque, $\mathrm{H}(\mathrm{q})$ is a symmetric and positive define inertia matrix, $\mathrm{B}(\mathrm{q})$ is the matrix of coriolios torques, $\mathrm{C}(\mathrm{q})$ is the matrix of centrifugal torques This is a decoupled system with simple second order linear differential dynamics. In other words, the component $\ddot{q}$ influences, with a double integrator relationship, only the variable $q_{i}$, independently of the motion of the other parts. Therefore, the angular acceleration is found as to be [1-11]:

$$
\ddot{\boldsymbol{q}}=\boldsymbol{H}^{-1}(\boldsymbol{q}) .\{\boldsymbol{\tau}-\{\boldsymbol{B}+\boldsymbol{C}\}\}
$$

This technique is very attractive fro m a control point of view.

Study of spherical motor is classified into two main groups: kinematics and dynamics. Calculate the relationship between rigid bodies and final part without any forces is called Kinematics. Study of this part is pivotal to design with an acceptable performance controller, and in real situations and practical applications. As expected the study of kinematics is divided into two main parts: forward and inverse kinematics. Forward kinematics has been used to find the position and orientation of task frame when angles of joints are known. Inverse kinematics has been used to find possible joints variable (angles) when all position and orientation of task frame be active [1].

According to the forward kinematics formulation;

$$
\boldsymbol{\Psi}(\boldsymbol{X}, \boldsymbol{q})=\mathbf{0}
$$

Where $\Psi(.) \in R^{n}$ is a nonlinear vector function, $X=\left[X_{1}, X_{2}, \ldots \ldots, X_{l}\right]^{T}$ is the vector of task space variables which generally task frame has three task space variables, three orientation, $q=\left[q_{1}, q_{2}, \ldots ., q_{n}\right]^{T}$ is a vector of angles or displacement, and finally $n$ is the number of actuated joints. The Denavit-Hartenberg (D-H) convention is a method of drawing spherical motor free body diagrams. Denvit-Hartenberg (D-H) convention study is necessary to calculate forward kinematics in this motor.

A systematic Forward Kinematics solution is the main target of this part. The first step to compute Forward Kinematics (F.K) is finding the standard D-H parameters. The following steps show the systematic derivation of the standard D-H parameters.

1. Locate the spherical motor

2. Label joints

3. Determine joint rotation $(\theta)$

4. Setup base coordinate frames.

5. Setup joints coordinate frames.

6. Determine $\alpha_{i}$, that $\alpha_{i}$, link twist, is the angle between $Z_{i}$ and $Z_{i+1}$.

7. Determine $d_{i}$ and $a_{i}$, that $a_{i}$, link length, is the distance between $Z_{i}$ and $Z_{i+1}$ along $X_{i} . d_{i}$, offset, is the distance between $X_{i-1}$ and $X_{i}$ along $Z_{i}$ axis.

8. Fill up the D-H parameters table. The second step to compute Forward kinematics is finding the rotation matrix $\left(R_{n}^{0}\right)$. The rotation matrix from $\left\{F_{i}\right\}$ to $\left\{F_{i-1}\right\}$ is given by the following equation;

$$
R_{i}^{i-1}=U_{i\left(\theta_{i}\right)} V_{i\left(\alpha_{i}\right)}
$$

Where $U_{i\left(\theta_{i}\right)}$ is given by the following equation [1-11];

$$
U_{i\left(\theta_{i}\right)}=\left[\begin{array}{ccc}
\cos \left(\theta_{i}\right) & -\sin \left(\theta_{i}\right) & 0 \\
\sin \left(\theta_{i}\right) & \cos \left(\theta_{i}\right) & 0 \\
0 & 0 & 1
\end{array}\right]
$$

and $V_{i\left(\alpha_{i}\right)}$ is given by the following equation [1-11];

$$
V_{i\left(\theta_{i}\right)}=\left[\begin{array}{ccc}
1 & 0 & 0 \\
0 & \cos \left(\alpha_{i}\right) & -\sin \left(\alpha_{i}\right) \\
0 & \sin \left(\alpha_{i}\right) & \cos \left(\alpha_{i}\right)
\end{array}\right]
$$

So $\left(R_{n}^{0}\right)$ is given by [8]

$$
R_{n}^{0}=\left(U_{1} V_{1}\right)\left(U_{2} V_{2}\right) \ldots \ldots\left(U_{n} V_{n}\right)
$$

The final step to compute the forward kinematics is calculate the transformation ${ }_{n}^{0} T$ by the following formulation [3]

$$
{ }_{n}^{0} T={ }_{1}^{0} T \cdot{ }_{2}^{1} T \cdot{ }_{3}^{2} T \ldots \ldots .{ }_{n}^{n-1} T=\left[\begin{array}{cc}
R_{n}^{0} & 0 \\
0 & 1
\end{array}\right]
$$

SLIDING MODE CONTROLLER: A significant challenge in control algorithms is a linear behavior controller design for nonlinear systems. When system works with various parameters and hard nonlinearities this technique is very useful in order to be implemented easily but it has some limitations such as working near the system operating point[12]. Some of nonlinear 
systems which work in industrial processes are controlled by linear PID controllers, but the design of linear controller for spherical motors are extremely difficult because they are nonlinear, uncertain and MIMO[33-55]. To reduce above challenges the nonlinear robust controllers is used to systems control. One of the powerful nonlinear robust controllers is sliding mode controller (SMC), although this controller has been analyzed by many researchers but the first proposed was in the 1950 [12-33].This controller is used in wide range areas such as in robotics, in control process, in aerospace applications and in power converters because it has an acceptable control performance and solve some main challenging topics in control such as resistivity to the external disturbance. The lyapunov formulation can be written as follows,

$$
V=\frac{1}{2} S^{T} . H . S
$$

The derivation of $V$ can be determined as,

$$
\dot{V}=\frac{1}{2} S^{T} \cdot \dot{H} \cdot S+S^{T} H \dot{S}
$$

The dynamic equation of spherical motor can be written based on the sliding surface as

$$
H \dot{S}=-V S+H \dot{S}+V S-\tau
$$

It is assumed that

$$
S^{T}(\dot{H}-2 V) S=0
$$

by substituting (11) in (10)

$$
\begin{aligned}
& \dot{V}=\frac{1}{2} S^{T} \dot{H} S-S^{T} V S+S^{T}(H \dot{S}+V S-\tau)= \\
& S^{T}(H \dot{S}+V S-\tau)
\end{aligned}
$$

Suppose the control input is written as follows

$$
\begin{aligned}
\widehat{\boldsymbol{\tau}}=\widehat{\boldsymbol{\tau}_{e q}}+\widehat{\boldsymbol{\tau}_{\text {dss }}=} & {\left[\widehat{H^{-1}}(\widehat{V})+\dot{S}\right] \widehat{H} } \\
& +K \cdot \operatorname{sgn}(S)+K_{v} S
\end{aligned}
$$

By replacing the equation (14) in (13)

$$
\begin{aligned}
& \dot{V}=S^{T}\left(H \dot{S}+V S-\widehat{H} \dot{S}-\widehat{V} S-K_{v} S-\right. \\
& K \operatorname{sgn}(S)=S^{T}\left(H \dot{S}+\widetilde{V} S-K_{v} S-\right. \\
& K \operatorname{sgn}(S))
\end{aligned}
$$

It is obvious that

$$
\left|\widetilde{H} \dot{S}+\widetilde{V} S-K_{v} S\right| \leq|\widetilde{H} \dot{S}|+|\widetilde{V} S|+\left|K_{v} S\right|
$$

The Lemma equation in spherical motor system can be written as follows

$$
\begin{aligned}
& K_{u}=\left[|\widetilde{H} \dot{S}|+|V S|+\left|K_{v} S\right|+\eta\right]_{i} \\
& , i=1,2,3,4, \ldots
\end{aligned}
$$

The equation (12) can be written as

$$
K_{u} \geq\left|\left[H \dot{S}+V S-K_{v} S\right]_{i}\right|+\eta_{i}
$$

Therefore, it can be shown that

$$
\dot{V} \leq-\sum_{i=1}^{n} \eta_{i}\left|S_{i}\right|
$$

Based on above discussion, the control law for spherical motor is written as:

$$
\boldsymbol{U}=\boldsymbol{U}_{\text {eq }}+\boldsymbol{U}_{\text {swith }}
$$

Where, the model-based component $\boldsymbol{U}_{\boldsymbol{e q}}$ is the nominal dynamics of systems and $\boldsymbol{U}_{\boldsymbol{e q}}$ can be calculate as follows:

$$
U_{e q}=\left[H^{-1}(B+C)+\dot{S}\right] H
$$

$\boldsymbol{U}_{\text {SWITCH }}$ is computed as;

$$
U_{\text {switch }}=K \cdot \operatorname{SGN}(\lambda e+\dot{e})
$$

by replace the formulation (22) in (20) the control output can be written as;

$$
U=U_{e q}+K \cdot S G N(S)
$$

By (23) and (21) the sliding mode control of spherical motor is calculated as;

$$
U=\left[H^{-1}(B+C)+\dot{S}\right] H+K \cdot \operatorname{SGN}(S)
$$

Figure 1 shows the conventional sliding mode controller for three dimension of spherical motor.

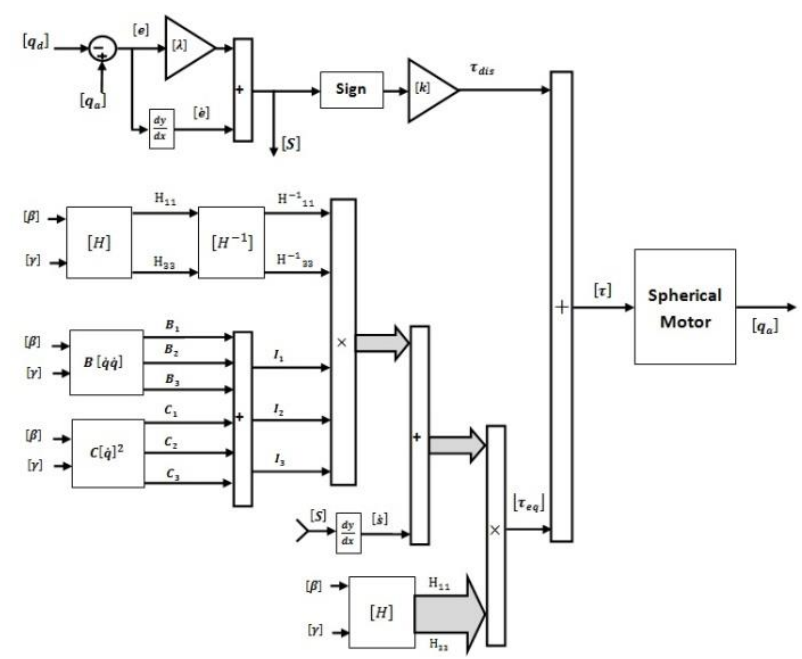

Fig. 1. Sliding Mode Control: 3 DOF spherical motor 
FUZZY LOGIC THEORY: This section provides a review about foundation of fuzzy logic based on [32- 53]. Supposed that $U$ is the universe of discourse and $x$ is the element of $U$, therefore, a crisp set can be defined as a set which consists of different elements $(x)$ will all or no membership in a set. A fuzzy set is a set that each element has a membership grade, therefore it can be written by the following definition;

$$
\boldsymbol{A}=\left\{\boldsymbol{x}, \boldsymbol{\mu}_{\boldsymbol{A}}(\boldsymbol{x}) \mid \boldsymbol{x} \in X\right\} ; \boldsymbol{A} \in \boldsymbol{U}
$$

Where an element of universe of discourse is $x, \mu_{A}$ is the membership function (MF) of fuzzy set. The me mbership function $\left(\mu_{A}(x)\right)$ of fuzzy set $A$ must have a value between zero and one. If the membership function $\mu_{A}(x)$ value equal to zero or one, this set change to a crisp set but if it has a value between zero and one, it is a fuzzy set. Defining membership function for fuzzy sets has divided into two main groups; namely; nu merical and functional method, which in numerical method each number has different degrees of membership function and functional method used standard functions in fuzzy sets. The membership function which is often used in practical applications includes triangular form, trapezoidal form, bell-shaped form, and Gaussian form.

Linguistic variable can open a wide area to use of fuzzy logic theory in many applications (e.g., control and system identification). In a natural artificial language all numbers replaced by words or sentences.

If - then Rule statements are used to formulate the condition statements in fuzzy logic. A single fuzzy If - then rule can be written by

\section{If $x$ is $A$ Then $y$ is $B$}

where $A$ and $B$ are the Linguistic values that can be defined by fuzzy set, the If - part of the part of " $x$ is $A$ " is called the antecedent part and the then part of the part of " $y$ is $B$ " is called the Consequent or Conclusion part. The antecedent of a fuzzy if-then rule can have multiple parts, which the following rules shows the multiple antecedent rules:

\section{if $e$ is $N B$ and $\dot{e}$ is ML then $T$ is $L L$}

where $e$ is error, $\dot{e}$ is change of error, $N B$ is Negative Big, $M L$ is Medium Left, $T$ is torque and $L L$ is Large Left. If - then rules have three parts, namely, fuzzify inputs, apply fuzzy operator and apply implication method which in fuzzify inputs the fuzzy statements in the antecedent replaced by the degree of membership, apply fuzzy operator used when the antecedent has multiple parts and replaced by single number between 0 to 1 , this part is a degree of support for the fuzzy rule, and apply implication method used in consequent of fuzzy rule to replaced by the degree of membership. The fuzzy inference engine offers a mechanis $m$ for transferring the rule base in fuzzy set which it is divided into two most important methods, namely, Mamdani method and
Sugeno method. Mamdani method is one of the common fuzzy inference systems and he designed one of the first fuzzy controllers to control of system engine. Mamdani's fuzzy inference system is divided into four major steps: fuzzification, rule evaluation, aggregation of the rule outputs and defuzzification. Michio Sugeno use a singleton as a membership function of the rule consequent part. The following definition shows the Mamdani and Sugeno fuzzy rule base

$$
\begin{aligned}
& \text { Mamdani F. } R^{1} \text { if } x \text { is A and } \\
& y \text { is } B \text { then } z \text { is } C \\
& \text { Sugeno F.R }{ }^{1} \text { if } \quad x \text { is } A \text { and } \\
& y \text { is } B \text { then } f(x, y) \text { is } C
\end{aligned}
$$

When $x$ and $y$ have crisp values fuzzification calculates the membership degrees for antecedent part. Rule evaluation focuses on fuzzy operation $(A N D / O R)$ in the antecedent of the fuzzy rules. The aggregation is used to calculate the output fuzzy set and several methodologies can be used in fuzzy logic controller aggregation, namely, Max-Min aggregation, Sum-Min aggregation, Max-bounded product, Max-drastic product, Max-bounded sum, Max-algebraic sum and Min-max. Two most common methods that used in fuzzy logic controllers are Max-min aggregation and Sum-min aggregation. Max-min aggregation defined as below

$$
\begin{aligned}
& \mu_{U}\left(x_{k}, y_{k}, U\right)=\mu_{\cup i=1}^{r} F R^{i}\left(x_{k}, y_{k}, U\right) \\
& =\max \left\{\min _{i=1}^{r}\left[\mu_{R}\left(x_{k}, y_{k}\right), \mu_{p_{m}}(U)\right]\right\}
\end{aligned}
$$

The Sum-min aggregation defined as below

$$
\begin{aligned}
& \mu_{U}\left(x_{k}, y_{k}, U\right)=\mu_{\cup U_{i=1}^{r} F R^{i}}\left(x_{k}, y_{k}, U\right) \\
& =\sum \min _{i=1}^{r}\left[\mu_{R_{p q}}\left(x_{k}, y_{k}\right), \mu_{p_{m}}(U)\right]
\end{aligned}
$$

where $r$ is the number of fuzzy rules activated by $x_{k}$ and $y_{k}$ and also $\mu_{\cup_{i=1}^{r} F R}\left(x_{k}, y_{k}, U\right)$ is a fuzzy interpretation of $i-t h$ rule. Defuzzification is the last step in the fuzzy inference system which it is used to transform fuzzy set to crisp set. Consequently defuzzification's input is the aggregate output and the defuzzification's output is a crisp number. Centre of gravity method ( $C O G)$ and Centre of area method $(C O A)$ are two most common defuzzification methods, which $C O G$ method used the following equation to calculate the defuzzification

$$
\operatorname{COG}\left(x_{k}, y_{k}\right)=\frac{\sum_{i} U_{i} \sum_{j=1}^{r} \cdot \mu_{u}\left(x_{k}, y_{k}, U_{i}\right)}{\sum_{i} \sum_{j=1}^{r} \cdot \mu_{u}\left(x_{k}, y_{k}, U_{i}\right)}
$$

and $C O A$ method used the following equation to calculate the defuzzification

$$
\operatorname{COA}\left(x_{k}, y_{k}\right)=\frac{\sum_{i} U_{i} \cdot \mu_{u}\left(x_{k}, y_{k}, U_{i}\right)}{\sum_{i} \mu_{U} \cdot\left(x_{k}, y_{k}, U_{i}\right)}
$$


Where $\operatorname{COG}\left(x_{k}, y_{k}\right)$ and $\operatorname{COA}\left(x_{k}, y_{k}\right)$ illustrates the crisp value of defuzzification output, $U_{i} \in U$ is discrete element of an output of the fuzzy set, $\mu_{U} \cdot\left(x_{k}, y_{k}, U_{i}\right)$ is the fuzzy set membership function, and $r$ is the number of fuzzy rules.

Based on foundation of fuzzy logic methodology; fuzzy logic controller has played important rule to design nonlinear controller for nonlinear and uncertain systems [53-66]. However the application area for fuzzy control is really wide, the basic form for all command types of controllers consists of;

- Input fuzzification (binary-to-fuzzy[B/F]conversion)

- Fuzzy rule base (knowledge base)

- Inference engine

- Output defuzzification binary $[\mathrm{F} / \mathrm{B}]$ conversion).

(fu zzy-to-

Figure 2 shows fuzzy controller operation.

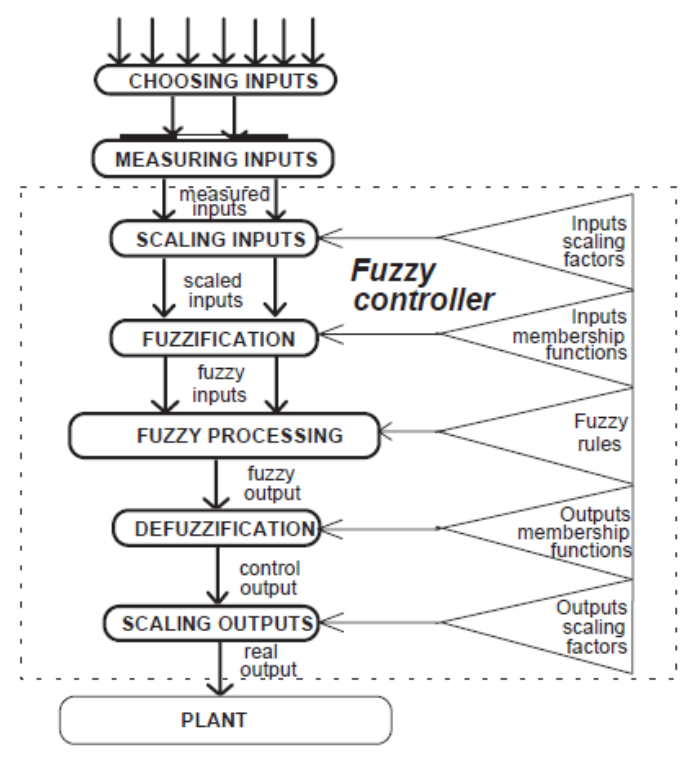

Fig. 2. Fuzzy Controller operation

\section{METHODOLOGY}

The methodology of boundary layer saturation method is achieve accurate tracking for non-linear and time varying system in presence of disturbance and parameter variations based on continuous feedback control law. To rectify the chattering phenomenon, the saturation continuous control is introduced. Figure $\mathbf{3}$ shows the linear saturation boundary layer function.

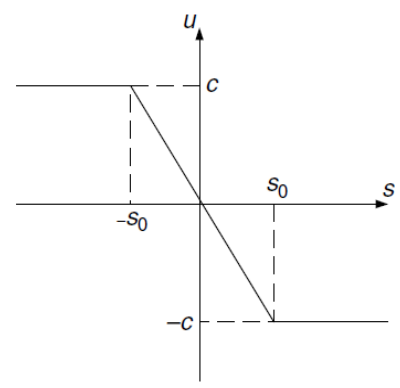

Fig. 3. Linear Saturation Boundary Layer Functions
Saturation boundary layer method has some disadvantages such as increase the error and reduces the speed of response. To solve linear boundary layer saturation challenge, design nonlinear intelligent saturation boundary layer function instead of linear saturation boundary method is introduced. This method is used to reduce or eliminate the chattering as well as reduce the error performance. However the design sliding mode fuzzy controller to reduce the chattering is faster and robust than sliding mode controller based on linear boundary layer method but adjust the fuzzy logic input and output gain updating factor is very difficult. Figure 4 shows the nonlinear artificial intelligence sliding mode controller based on fuzzy logic methodology.

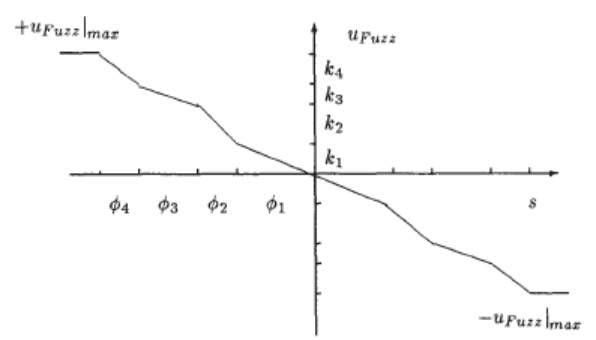

Fig. 4. Nonlinear Fuzzy Saturation Boundary Layer Functions

To reduce the chattering phenomenon, this research is focused on applied parallel fuzzy logic theorem in discontinuous part of sliding mode controller as a switching compensator. Fuzzy logic theory is used in parallel with switching part of sliding mode controller to compensate the limited uncertainty in system's dynamic and its impact on the chattering phenomenon. In this method fuzzy logic theorem is applied to switching sliding mode controller to estimate the challenge of switching part in chattering based on nonlinear switching function. To achieve this goal, the switching function of pure sliding mode controller is parallel estimated by Mamdani's performance/ error-based fuzzy logic methodology. This technique was employed to obtain the desired control behavior with a number of information about dynamic model of system and a fuzzy switching control was applied to reinforce system performance. Reduce or eliminate the chattering phenomenon and reduce the error are played important role, therefore switching method is used beside the artificial intelligence part to solve the chattering problem with respect to reduce the error. Figure 5 shows the intelligent chattering free sliding mode controller for three dimensions spherical motor.

Equivalent part of sliding mode controller is based on nonlinear dynamic formulations of spherical motor. Spherical motor's dynamic formulations are highly nonlinear and some of parameters are unknown therefore design a controller based on dynamic formulation is complicated. In a typical PD method, the controller corrects the error between the desired input value and the measured value. Since the actual position is the measured signal. The derivative part of PD methodology is worked based on change of error and the derivative coefficient. Based on the SMC controller; 


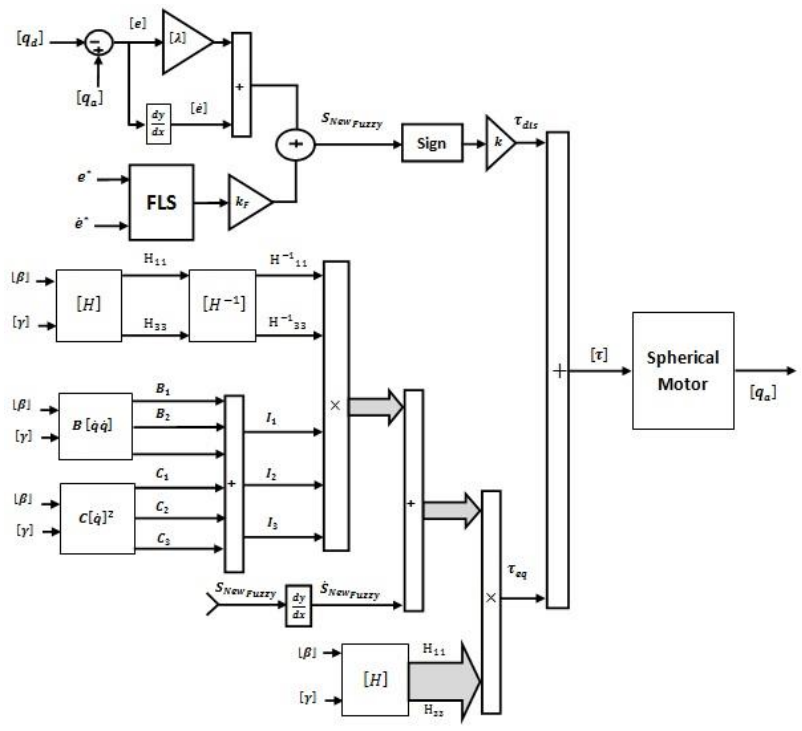

Fig 5 New chattering free intelligent sliding mode controller

$$
\begin{aligned}
& \boldsymbol{\tau}_{\text {new-d } t s}=K \cdot \operatorname{sgn}\left(S_{\text {new-fuzzy }}\right) \\
& \boldsymbol{S}_{\text {new-fuzzy }} \triangleq \lambda \boldsymbol{e}+\dot{\boldsymbol{e}}+\mathrm{U}_{\text {fuzzy }}=\lambda \boldsymbol{e}+\dot{\boldsymbol{e}}+ \\
& \frac{\sum_{\mathrm{l}=1}^{\mathrm{M}} \theta^{\mathrm{l}}\left[\prod_{\mathrm{i}=1}^{\mathrm{n}} \exp \left(-\left(\frac{\mathrm{x}_{\mathrm{j}}-\alpha_{\mathrm{i}}^{\mathrm{l}}}{\delta_{\mathrm{i}}^{\mathrm{l}}}\right)^{2}\right)\right]}{\sum_{\mathrm{l}=1}^{\mathrm{M}}\left[\prod_{\mathrm{i}=1}^{\mathrm{n}} \exp \left(-\left(\frac{\mathrm{x}_{\mathrm{i}}-\alpha_{\mathrm{i}}^{\mathrm{l}}}{\delta_{\mathrm{i}}^{\mathrm{l}}}\right)^{2}\right)\right]}
\end{aligned}
$$

This is suitable for real-time control applications when powerful processors, which can execute complex algorith ms rapidly, are not accessible. In this theory the behavior and dynamic of fuzzy logic compensator is defined by rule base. However defined and number of rule base play important role to design high quality estimator but system has limitation to the number of rule base to implementation and the speed of response. In PD like fuzzy controller error and change of error are the inputs and if any input is described with seven linguistic values, and any rule has two conditions we will need $7 \times$ $7=49$ rules. Table 1 shows the rule table of PD like fuzzy controller based on seven linguistic variables for inputs

\begin{tabular}{|c|c|c|c|c|c|c|c|}
\hline$e^{\Delta e}$ & PB & PM & PS & $\mathbf{Z}$ & NS & NM & NB \\
\hline PB & NB & NB & NB & NB & NM & NS & Z \\
\hline PM & NB & NB & NB & NM & NS & $\mathbf{Z}$ & PS \\
\hline PS & NB & NB & NM & NS & $\mathbf{Z}$ & PS & $\overline{P M}$ \\
\hline Z & NB & NM & NS & $\mathbf{Z}$ & PS & PM & PB \\
\hline NS & NM & NS & $\mathbf{Z}$ & PS & PM & PB & PB \\
\hline NM & NS & $\mathbf{Z}$ & PS & PM & PB & PB & PB \\
\hline NB & $\mathbf{Z}$ & PS & PM & PB & PB & PB & PB \\
\hline
\end{tabular}
and totally 49 rules.

Table 1. Rule Table of PD like Fuzzy Controller

This table includes 49 rules. We are taking into account now not just the error but the change-of-error as well. It allows describing the dynamics of the controller. To explain how this rules set works and how to choose the rules, let us divide the set of all rules into the following five groups:

Group 1: In this group of rules both $\boldsymbol{e}$ and $\boldsymbol{\Delta} \boldsymbol{e}$ are (positive or negative) small or zero. This means that the current value of the process output variable has deviated from the desired level (the set-point) but is still close to it. Because of this closeness the control signal should be zero or small in magnitude and is intended to correct small deviations from the set-point. Therefore, the rules in this group are related to the steady-state behavior of the process. The change-of-error, when it is Negative Small or Positive Small, shifts the output to negative or positive region, because in this case, for example, when $\boldsymbol{e}(\boldsymbol{t})$ and $\boldsymbol{\Delta} \boldsymbol{e}(\boldsymbol{t})$ are both Negative Small the error is already negative and, due to the negative change-of-error, tends to become more negative. To prevent this trend, one needs to increase the magnitude of the control output.

Group 2: For this group of rules $\boldsymbol{e}(\boldsymbol{t})$ is Positive Big or Medium which implies that actual input is significantly above the set point. At the same time since $\boldsymbol{\Delta} \boldsymbol{e}(\boldsymbol{t})$ is negative, this means that actual input is moving towards the set-point. The control signal is intended to either speed up or slow down the approach to the setpoint. For example, if actual input is much below the setpoint $(\boldsymbol{e}(\boldsymbol{t})$ is Positive Big) and it is moving towards the set-point with a small step $(\boldsymbol{\Delta e}(\boldsymbol{t})$ is Negative Small) then the magnitude of this step has to be significantly increased ( $\boldsymbol{U}$ is Negative Medium). However, when actual input is still much below the set-point $(\boldsymbol{e}(\boldsymbol{t})$ is Positive Big) but it is moving towards the set-point very fast $(\boldsymbol{\Delta} \boldsymbol{e}(\boldsymbol{t})$ is Negative Big) no control action can be recommended because the error will be compensated due to the current trend.

Group 3: For this group of rules actual output is either close to the set-point $(\boldsymbol{e}(\boldsymbol{t})$ is Positive Small, Zero, Negative Small) or significantly above it (Negative Medium, Negative Big). At the same time, since $\Delta \boldsymbol{e}(\boldsymbol{t})$ is negative, actual input is moving away from the setpoint. The control here is intended to reverse this trend and make actual input, instead of moving away from the set-point, start moving towards it. So here the main reason for the control action choice is not just the current error but the trend in its change.

Group 4: For this group of rules $\boldsymbol{e}(\boldsymbol{t})$ is Negative Medium or Big, which means that actual input is significantly below the set-point. At the same time, since $\boldsymbol{\Delta} \boldsymbol{e}(\boldsymbol{t})$ is positive, actual input is moving towards the setpoint. The control is intended to either speed up or slow down the approach to the set-point. For example, if actual input is much above the set-point $(\boldsymbol{e}(\boldsymbol{t})$ is Negative Big) and it is moving towards the set-point with a somewhat large step ( $\boldsymbol{\Delta} \boldsymbol{e}(\boldsymbol{t})$ is Positive Medium), then the magnitude of this step has to be only slightly enlarged (output is Negative Small).

Group 5: The situation here is similar to the Group 3 in some sense. For this group of rules $\boldsymbol{e}(\boldsymbol{t})$ is either close to the set-point (Positive Small, Zero, Negative Small) or significantly above it (Positive Medium, Positive Big). 
At the same time since $\boldsymbol{\Delta e}(\boldsymbol{t})$ is positive actual input is moving away from the set-point. This control signal is intended to reverse this trend and make actual input instead of moving away from the set-point start moving towards it. The PD like fuzzy controller shows in Figure 6.

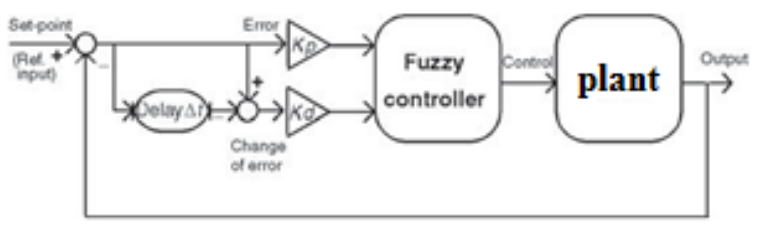

Fig. 6. Block diagram of PD like Fuzzy Controller

Based on literature, SMC formulation is written by;

$$
\begin{aligned}
\tau=\left[H^{-1}(B+C)\right. & +\dot{S}] H+K \\
\cdot S G N & \left(S_{\text {new-fuzzy }}\right)
\end{aligned}
$$

The second challenge in this research is the role of nonlinearity term in presence of uncertainty and external disturbance. To solve this challenge artificial intelligence based controller is introduce. This type of controller is intelligent therefore design a dynamic of system based on experience knowledge is done by this method. However defined and number of rule base play important role to design high quality controller but system has limitation to the number of rule base to implementation and the speed of response. The parallel fuzzy error-based compensator of sliding mode controller's output is written;

$$
\hat{\boldsymbol{\tau}}=\tau_{e q_{f u z z y}}+\tau_{S M C}
$$

Based on fuzzy logic methodology

$$
f(x)=U_{f u z z y}=\sum_{l=1}^{M} \theta^{T} \zeta(x)
$$

where $\boldsymbol{\theta}^{\boldsymbol{T}}$ is adjustable parameter (gain updating factor) and $\boldsymbol{\zeta}(\boldsymbol{x})$ is defined by;

$$
\zeta(x)=\frac{\sum_{i} \mu\left(x_{i}\right) x_{i}}{\sum_{i} \mu\left(x_{i}\right)}
$$

Design an error-based parallel fuzzy compensate of equivalent part based on Mamdani's fuzzy inference method has four steps, namely, fuzzification, fu zzy rule base and rule evaluation, aggregation of the rule output (fuzzy inference system) and defuzzification. This part of controller has two inputs (error and change of error) and one output (fuzzy torque estimator). This controller is worked based on table 1 and 49 rule bases. Figure 7 shows parallel mode PD like fuzzy sliding mode controller for three dimension spherical motor.

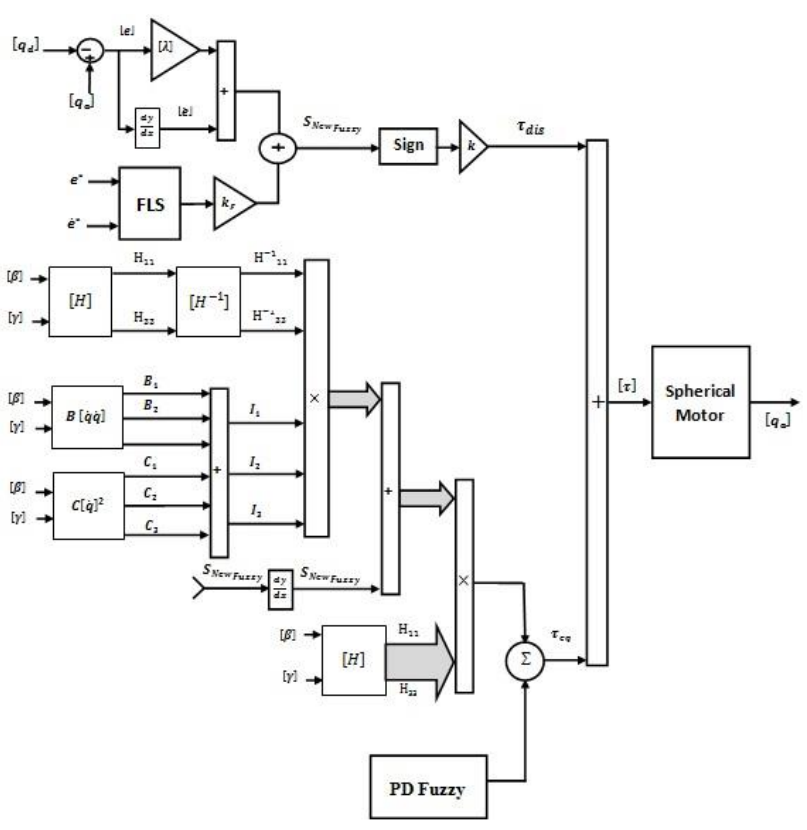

Fig. 7. Block diagram of intelligent chattering free PD like fuzzy sliding mode controller

\section{RESUlT AND DisCUSSION}

Modified fuzzy chattering free PD like fuzzy sliding mode controller is implemented in MATLAB/ SIMULINK environment. Tracking performance and disturbance rejections are compared for step trajectory.

Tracking performances: In proposed controller; the performance is depended on three important parameters; nonlinear equivalent part, and PD like fuzzy controller. According to above discussion, however modified fuzzy chattering free PD like fuzzy sliding mode controller have accept performance in certain parameters but pure sliding mode controller have overshoot about $1 \%$ and steady state error is about 0.05. Based on Fig 8, pure sliding mode controller can eliminate the chattering but it has steady state error. To solve this challenge the output gain updating factor of proposed controller is decreased. In this design rise time in pure sliding mode controller is lower than proposed method.

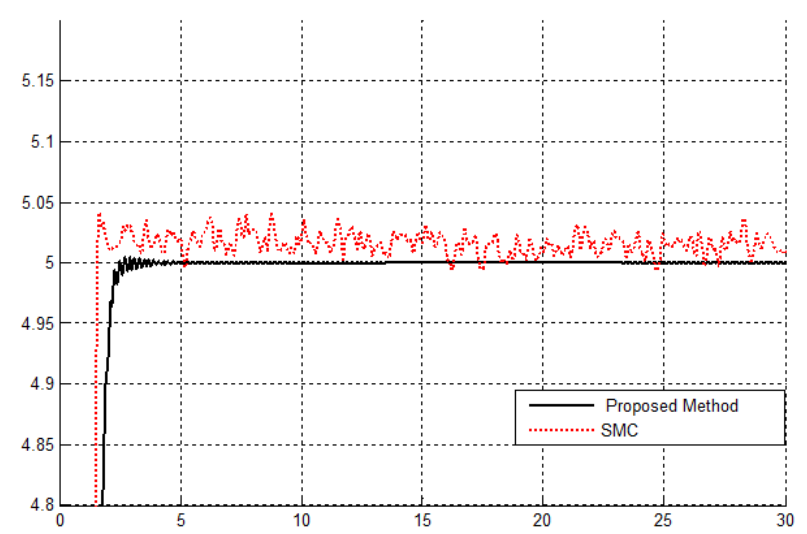

Fig. 8. Sliding mode controller and proposed method 
Disturbance rejection: Figure 9 shows the power disturbance elimination in proposed method and pure sliding mode controller in presence of external disturbance and uncertainty parameters. The disturbance rejection is used to test and analyzed the robustness comparisons of these controllers for step trajectory. A band limited white noise with predefined of $40 \%$ the power of input signal value is applied to the step trajectory. According to the following graph, pure sliding mode controller has moderate fluctuation in presence of external disturbance and uncertainty.

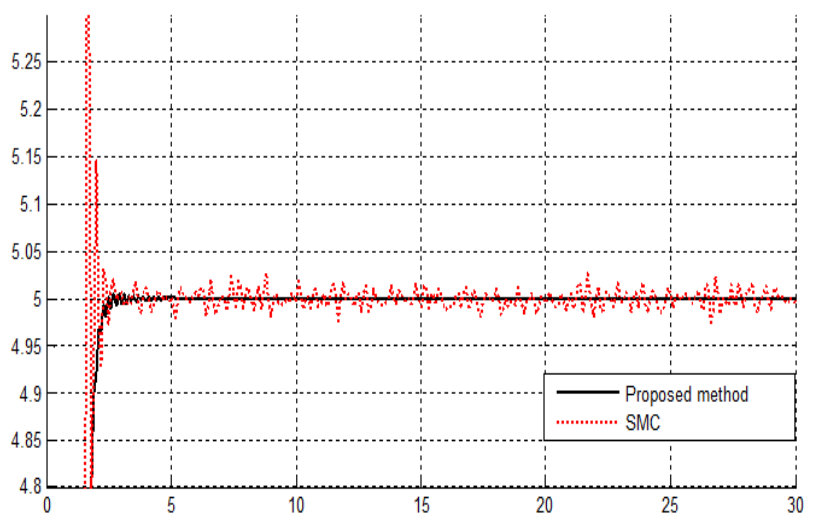

Fig. 9. Sliding Mode controller and proposed method in presence of external disturbance

Based on above graph, pure sliding mode controller has many challenges in presence of external disturbance. To eliminate above challenge, PD like fuzzy controller is used to eliminate the chattering and fuzzy estimator is used to estimate uncertainty. The PD like fuzzy controller can modify the sliding mode controller based on the following important notes;

- Decrease the output scaling factor of the PD-part

- Increase the scaling factor for an integral input compared to other inputs

- Apply the centre of gravity defuzzification method

- Reduce the width of the membership function for the zero class of the error signal and Redistribute the membership functions, increasing their concentration around the zero point.

\section{CONCLUSION}

According to the dynamic information in spherical motor, this system is highly nonlinear and MIMO. Control of this system is complicated; sliding mode controller is robust, stable and reliable controller and is the best alternative in this system. conventional sliding mode controller has two main challenges; chattering phenomenon and dynamic nonlinear equivalent part. PD like intelligent theory based on fuzzy logic is used to reduce the chattering. This design can eliminate the chattering based on 49 Mamdani rule bases. To re solve the nonlinearity challenge in presence of uncertainty, parallel intelligent method is used based on PD like fuzzy theory and 49 rule bases. According to the results; proposed controller has suitable control performance.

\section{ACKNOWLEDGMENT}

The authors would like to thank the anonymous reviewers for their careful reading of this paper and for their helpful comments. This work was supported by the SSP Institute of Advance Science and Technology Program of Iran under grant no. 2013-Persian Gulf-2A.

\section{REFERENCES}

[1] Vachtsevanos, G. I., Davey, K. and Lee, K. M., "Development of a Novel Intelligent Robotic Manipulator," IEEE Control Sy stem Magazine, 1987, pp.915.

[2] Davey, K., Vachtsevanos, G. I., and Powers, R., "An analysis of Fields and Torques in Spherical Induction Motors," IEE Transactions on Magnetics, Vol. MAG-23, 1987, pp. 273-282.

[3] Foggia, A., Oliver, E., Chappuis, F., "New Three Degrees of Freedom Electromagnetic Actuator," Conference Record -lAS Annual Meeting, Vol. 35, New York, 1988.

[4] Lee, K. M., Vachtsevanos, G. and Kwan, C-K., "Development of a Spherical Wrist Stepper Motor," Proceedings of the 1988 IEEE Intemational Conference on Robotics and Automation, Philadelphia, PA. April 26-29.

[5] Lee, K. M., Pei. I., "Kinematic Analysis of a Three Degree-of-Freedom Spherical Wrist Actuator," The Fifth International Conference on Advanced Robotics, Italy, 1991.

[6] Wang, I., Jewel, G., Howe, D., "Modeling of a Novel Spherical Pennanent Magnet Actuator," Proceedings of IEEE International Conference on Robotics and Automation, Albuquerque, New Mexico, pp 1190-1195, 1997.

[7] Wang, I., Jewel, G., Howe, D., "Analysis, Design and Control of a Novel Spherical Pennanent Magnet Actuator," lEE Proceedings on Electrical Power Applications., vol. 154, no. 1, 1998.

[8] Chirikjian, G. S., and Stein, D., "Kinematic Design and Commutation of a Spherical Stepper Motor," IEEEIASME Transactions on Mechatronics, vol. 4, n 4, Piscataway, New Jersey, pp. 342-353, Dec. 1999.

[9] Kahlen, K., and De Doncker, R. W., "CW'l'ent Regulators for Multi-phase Pennanent Magnet Spherical Machines." Industry Applications Conference Record of the 2000 IEEE, vol. 3, 2000, pp. 2011-2016.

[10] Lee, K. M., Pei, I., and Gilboa, U., "On the Development of a Spherical Wrist Actuator," Proceedings of the 16th NSF Conference on Manufacturing Systems Research, Tempe AZ, January 8-12, 1990.

[11] Yang, C., Back, Y. S., "Design and Control of the 3dcgn:es of freedom actuator by Controlling the Electromagnetic Force," IEEE Transactions on Magnetics, May, 1999, pp.3607-3609.

[12] Samira Soltani \& Farzin Piltan, "Design Artificial Nonlinear Controller Based on Computed Torque like Controller with Tunable Gain", World Applied Science Journal (WASJ), 14 (9): 1306-1312, 2011.

[13] Farzin Piltan, Mohammadali Dialame, Abbas Zare \& Ali Badri,"Design Novel Lookup Table Changed Auto Tuning FSMC:Applied to Robot Manipulator", International Journal of Engineering, 6 (1):25-41, 2012

[14] Farzin Piltan, Mohammad Keshavarz, Ali Badri \& Arash Zargari,"Design Novel Nonlinear Controller Applied to RobotManipulator: Design New Feedback Linearization 
Fuzzy Controller with Minimum Rule Base Tuning Method", International Journal of Robotics and Automation,3 (1):1-12, 2012

[15] Farzin Piltan, Iman Nazari, Sobhan Siamak, Payman Ferdosali,"Methodology of FPGA-Based Mathematical error-Based Tuning Sliding Mode Controller", International Journal of Control and Automation, 5(1), 89118, 2012

[16] Farzin Piltan, Bamdad Boroomand, Arman Jahed \& Hossein Rezaie, "Methodology of Mathematical ErrorBased Tuning Sliding Mode Controller", International Journal of Engineering, 6 (2):96-117, 2012

[17] Farzin Piltan, Sara Emamzadeh, Zahra Hivand, Fatemeh Shahriyari \& Mina Mirazaei, "PUMA-560 Robot Manipulator Position Sliding Mode Control Methods Using MATLAB/SIMULINK and Their Integration into Graduate/Undergraduate Nonlinear Control, Robotics and MATLAB Courses", International Journal of Robotics and Automation, 3(3):106-150, 2012

[18] Farzin Piltan, Ali Hosainpour, Ebrahim Mazlomian, Mohammad Shamsodini, Mohammad H. Yarmahmoudi, "Online Tuning Chattering Free Sliding Mode Fuzzy Control Design: Lyapunov Approach", International Journal of Robotics and Automation, 3(3):77105, 2012

[19] Farzin Piltan, Mina Mirzaei, Forouzan Shahriari, Iman Nazari, Sara Emamzadeh, "Design Baseline Computed Torque Controller", International Journal of Engineering, 6(3): 129-141, 2012

[20] Farzin Piltan, Mohammad H. Yarmahmoudi, Mohammad Shamsodini, Ebrahim Mazlomian, Ali Hosainpour, "PUMA-560 Robot Manipulator Position Computed Torque Control Methods Using MATLAB/SIMULINK and Their Integration into Graduate Nonlinear Control and MATLAB Courses", International Journal of Robotics and Automation, 3(3): 167-191, 2012

[21] Farzin Piltan, Hossein Rezaie, Bamdad Boroomand, Arman Jahed, "Design Robust Backstepping on-line Tuning Feedback Linearization Control Applied to IC Engine", International Journal of Advance Science and Technology, 11:40-22, 2012

[22] Farzin Piltan, Mohammad R. Rashidian, Mohammad Shamsodini and Sadeq Allahdadi, Effect of Rule Base on the Fuzzy-Based Tuning Fuzzy Sliding Mode Controller: Applied to $2^{\text {nd }}$ Order Nonlinear System", International Journal of Advanced Science and Technology, 46:39-70, 2012

[23] Farzin Piltan, Arman Jahed, Hossein Rezaie and Bamdad Boroomand, "Methodology of Robust Linear On-line High Speed Tuning for Stable Sliding Mode Controller: Applied to Nonlinear System", International Journal of Control and Automation, 5(3): 217-236, 2012

[24] Farzin Piltan, Bamdad Boroomand, Arman Jahed and Hossein Rezaie, "Performance-Based Adaptive Gradient Descent Optimal Coefficient Fuzzy Sliding Mode Methodology", International Journal of Intelligent Sy stems and Applications, , vol.4, no.11, pp.40-52, 2012.

[25] Farzin Piltan, Mehdi Akbari, Mojdeh Piran , Mansour Bazregar, "Design Model Free Switching Gain Scheduling Baseline Controller with Application to Automotive Engine", International Journal of Information Technology and Computer Science, vol.5, no.1, pp.65-73, 2013.DOI: 10.5815/ijitcs.2013.01.07.

[26] Farzin Piltan, Mojdeh Piran , Mansour Bazregar, Mehdi Akbari, "Design High Impact Fuzzy Baseline Variable Structure Methodology to Artificial Adjust Fuel Ratio",
International Journal of Intelligent Systems and Applications, vol.5, no.2, pp.59-70, 2013. DOI: 10.5815/ijisa.2013.02.07.

[27] Farzin Piltan, M. Bazregar, M. kamgari, M. Akbari and M. Piran, "Adjust the Fuel Ratio by High Impact Chattering Free Sliding Methodology with Application to Automotive Engine", International Journal of Hybrid Information Technology, 6(1), 2013.

[28] Farzin Piltan, S. Zare , F. ShahryarZadeh, M. Mansoorzadeh, M. kamgari, "Supervised Optimization of Fuel Ratio in IC Engine Based on Design Baseline Computed Fuel Methodology", International Journal of Information Technology and Computer Science, vol.5, no.4, pp.76-84, 2013.DOI: 10.5815/ijitcs.2013.04.09.

[29] Farzin Piltan, M. Mansoorzadeh, S. Zare, F.Shahry arzadeh, M. Akbari, "Artificial Tune of Fuel Ratio: Design a Novel SISO Fuzzy Backstepping Adaptive Variable Structure Control", International Journal of Electrical and Computer Engineering, 3(2), 2013.

[30] M. Bazregar, Farzin Piltan, A. Nabaee and M.M. Ebrahimi, "Parallel Soft Computing Control Optimization Algorithm for Uncertainty Dynamic Systems", International Journal of Advanced Science and Technology, 51, 2013.

[31] Farzin Piltan, M.H. Yarmahmoudi, M. Mirzaei, S. Emamzadeh, Z. Hivand, "Design Novel Fuzzy Robust Feedback Linearization Control with Application to Robot Manipulator", International Journal of Intelligent Systems and Applications, vol.5, no.5, pp.1-10, 2013.DOI: 10.5815/ijisa.2013.05.01

[32] Sh. Tayebi Haghighi, S. Soltani, Farzin Piltan, M. kamgari, S. Zare, "Evaluation Performance of IC Engine: Linear Tunable Gain Computed Torque Controller Vs. Sliding Mode Controller", International Journal of Intelligent Systems and Applications, vol.5, no.6, pp.78-88, 2013.DOI: 10.5815/ijisa.2013.06.10.

[33] Amin Jalali, Farzin Piltan, M. Keshtgar, M. Jalali, "Colonial Competitive Optimization Sliding Mode Controller with Application to Robot Manipulator", International Journal of Intelligent Systems and Applications, vol.5, no.7, pp.50-56, 2013. DOI: 10.5815/ijisa.2013.07.07.

[34] Salehi, Farzin Piltan, M. Mousavi, A. Khajeh, M. R. Rashidian, "Intelligent Robust Feed-forward Fuzzy Feedback Linearization Estimation of PID Control with Application to Continuum Robot", International Journal of Information Engineering and Electronic Business, vol.5, no.1, pp.1-16,2013. DOI: 10.5815/ijieeb.2013.01.01.

[35] Farzin Piltan, M.J. Rafaati, F. Khazaeni, A. Hosainpour, S. Soltani, “A Design High Impact Lyapunov Fuzzy PD-PlusGravity Controller with Application to Rigid Manipulator", International Journal of Information Engineering and Electronic Business, vol.5, no.1, pp.17-25, 2013. DOI: 10.5815/ijieeb.2013.01.02.

[36] Amin Jalali, Farzin Piltan, A. Gavahian, M. Jalali, M. Adibi, "Model-Free Adaptive Fuzzy Sliding Mode Controller Optimized by Particle Swarm for Robot manipulator", International Journal of Information Engineering and Electronic Business, vol.5, no.1, pp.68-78, 2013. DOI: 10.5815/ijieeb.2013.01.08.

[37] Farzin Piltan, F. ShahryarZadeh ,M. Mansoorzadeh ,M. kamgari, S. Zare, "Robust Fuzzy PD Method with Parallel Computed Fuel Ratio Estimation Applied to Automotive Engine", International Journal of Intelligent Systems and Applications, vol.5, no.8, pp.83-92, 2013. DOI: 10.5815/ijisa.2013.08.10.

[38] Farzin Piltan, A. Nabaee, M.M. Ebrahimi, M. Bazregar, "Design Robust Fuzzy Sliding Mode Control Technique 
for Robot Manipulator Systems with Modeling Uncertainties", International Journal of Information Technology and Computer Science, vol.5, no.8, pp.123135, 2013. DOI: 10.5815/ijitcs.2013.08.12.

[39] Farzin Piltan, M. Mansoorzadeh, M. Akbari, S. Zare, F. ShahryarZadeh "Management of Environmental Pollution by Intelligent Control of Fuel in an Internal Combustion Engine" Global Journal of Biodiversity Science And Management, 3(1), 2013.

[40] M. M. Ebrahimit Farzin Piltan, M. Bazregar and A.R. Nabaee, "Intelligent Robust Fuzzy-Parallel Optimization Control of a Continuum Robot Manipulator", International Journal of Control and Automation, 6(3), 2013.

[41] O.R. Sadrnia, Farzin Piltan, M. Jafari, M. Eram and M. Shamsodini, "Design PID Estimator Fuzzy plus Backstepping to Control of Uncertain Continuum Robot", International Journal of Hybrid Information Technology, 6(4), 2013.

[42] AminJalali, Farzin Piltan, H. Hashemzadeh, A. Hasiri, M.R Hashemzadeh, "Design Novel Soft Computing Backstepping Controller with Application to Nonlinear Dynamic Uncertain System", International Journal of Intelligent Systems and Applications, vol.5, no.10, pp.93105, 2013. DOI: 10.5815/ijisa.2013.10.12.

[43] M. Moosavi, M. Eram, A. Khajeh, O. Mahmoudi and Farzin Piltan, "Design New Artificial Intelligence Base Modified PID Hybrid Controller for Highly Nonlinear System", International Journal of Advanced Science and Technology, 57, 2013.

[44] S. Zahmatkesh, Farzin Piltan, K. Heidari, M. Shamsodini, S. Heidari, "Artificial Error Tuning Based on Design a Novel SISO Fuzzy Backstepping Adaptive Variable Structure Control" International Journal of Intelligent Systems and Applications, vol.5, no.11, pp.34-46, 2013. DOI: 10.5815/ijisa.2013.11.04.

[45] S. Heidari, Farzin Piltan, M. Shamsodini, K. Heidari and S. Zahmatkesh, "Design New Nonlinear Controller with Parallel Fuzzy Inference System Compensator to Control of Continuum Robot Manipulator",International Journal of Control and Automation, 6(4), 2013.

[46] FarzinPiltan, M. Kamgari, S. Zare, F. Shahry arZadeh, M Mansoorzadeh, "Design Novel Model Reference Artificial Intelligence Based Methodology to Optimized Fuel Ratio in IC Engine", International Journal of Information Engineering and Electronic Business, vol.5, no.2, pp.44-51, 2013. DOI: 10.5815/ijieeb.2013.02.07.

[47] Farzin Piltan, Mehdi Eram, Mohammad Taghavi, Omid Reza Sadrnia, Mahdi Jafari,"Nonlinear Fuzzy Model-base Technique to Compensate Highly Nonlinear Continuum Robot Manipulator", IJISA, vol.5, no.12, pp.135-148, 2013. DOI: $10.5815 /$ ijisa.2013.12.12

[48] Amin Jalali, Farzin Piltan, Mohammadreza Hashemzadeh, Fatemeh Bibak Varavi, Hossein Hashemzadeh,"Design Parallel Linear PD Compensation by Fuzzy Sliding Compensator for Continuum Robot", IJTCS, vol.5, no.12, pp.97-112, 2013. DOI: 10.5815/ijitcs.2013.12.12

[49] Farzin Piltan, A. Hosainpour, S. Emamzadeh, I. Nazari, M. Mirzaie, "Design Sliding Mode Controller of with Parallel Fuzzy Inference System Compensator to Control of Robot Manipulator", International Journal of Robotics and Automation, Vol. 2, No. 4, December 2013, pp. 149 162.

[50] Farzin Piltan, Mahdi Jafari, Mehdi Eram, Omid Mahmoudi, Omid Reza Sadrnia, "Design Artificial Intelligence-Based Switching PD plus Gravity for Highly Nonlinear Second Order System", International Journal of Engineering and Manufacturing, vol.3, no.1, pp.38-57, 2013.DOI: 10.5815/ijem.2013.01.04

[51] Farzin Piltan, Sara Emamzadeh, Sara Heidari, Samaneh Zahmatkesh, Kamran Heidari, "Design Artificial Intelligent Parallel Feedback Linearization of PID Control with Application to Continuum Robot", International Journal of Engineering and Manufacturing, vol.3, no.2, pp.51-72, 2013.DOI: 10.5815/ijem.2013.02.04

[52] Mohammad Mahdi Ebrahimi, Farzin Piltan, Mansour Bazregar, AliReza Nabaee,"Artificial Chattering Free online Modified Sliding Mode Algorithm: Applied in Continuum Robot Manipulator", International Journal of Information Engineering and Electronic Business, vol.5, no.5, pp.57-69, 2013. DOI: 10.5815/ijieeb.2013.05.08

[53] Arman Jahed, Farzin Piltan, Hossein Rezaie, Bamdad Boroomand, "Design Computed Torque Controller with Parallel Fuzzy Inference System Compensator to Control of Robot Manipulator", International Journal of Information Engineering and Electronic Business, vol.5, no.3, pp.66-77, 2013. DOI: 10.5815/ijieeb.2013.03.08

[54] Mohammad Shamsodini, Farzin Piltan, Mahdi Jafari, Omid reza Sadrnia, Omid Mahmoudi,"Design Modified Fuzzy Hy brid Technique: Tuning By GDO", IJMECS, vol.5, no.8, pp.58-72, 2013.DOI: 10.5815/ijmecs.2013.08.07

[55] Mahdi Mirshekaran, Farzin Piltan,Zahra Esmaeili, Tannaz Khajeaian, Meysam Kazeminasab,"Design Sliding Mode Modified Fuzzy Linear Controller with Application to Flexible Robot Manipulator", IJMECS, vol.5, no.10, pp.53-63, 2013.DOI: 10.5815/ijmecs.2013.10.07

[56] Meysam Kazeminasab, Farzin Piltan, Zahra Esmaeili, Mahdi Mirshekaran, Alireza Salehi ,"Design Parallel Fuzzy Partly Inverse Dynamic Method plus Gravity Control for Highly Nonlinear Continuum Robot", IJISA, vol.6, no.1, pp.112-123, 2014. DOI: 10.5815/ijisa.2014.01.12.

[57] Mansour Bazregar, Farzin Piltan, Mehdi Akbari, Mojdeh Piran,"Management of Automotive Engine Based on Stable Fuzzy 10.5815/ijitcs.2014.01.12.

[58] Alireza Siahbazi, Ali Barzegar, Mahmood Vosoogh, Abdol Majid Mirshekaran, Samira Soltani,"Design Modified Sliding Mode Controller with Parallel Fuzzy Inference System Compensator to Control of Spherical Motor", IJISA, vol.6, no.3, pp.12-25, 2014. DOI: 10.5815/ijisa.2014.03.02

[59] Narges Gholami Mozafari, Farzin Piltan, Mohammad Shamsodini, Azita Yazdanpanah, Ali Roshanzamir,"On Line Tuning Premise and Consequence FIS Based on Lyaponuv Theory with Application to Continuum Robot", IJISA, vol.6, no.3, pp.96-110, 2014. DOI: 10.5815/ijisa.2014.03.10.

[60] Mansour Bazregar, Farzin Piltan, AliReza Nabaee, MohammadMahdi Ebrahimi,"Design Modified Fuzzy PD Gravity Controller with Application to Continuum Robot", IJITCS, vol.6, no.3, pp.82-94, 2014. DOI: $10.5815 /$ ijitcs.2014.03.10

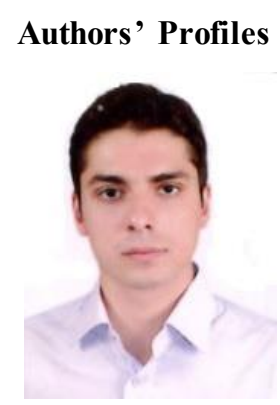

and spherical motor.
Mohammad Ali Tayebi is currently working as a co researcher in Control and Robotic $\mathrm{Lab}$ at the institute of advance science and technology, IRAN SSP research and development Center. $\mathrm{He}$ is a Master in field of Control engineering from Azad University, IRAN. His current research interests are in the area of nonlinear control, artificial control system and robotics, 


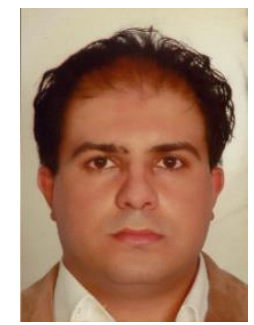

Farzin Piltan was born on 1975, Shiraz, Iran. In 2004 he is jointed Institute of Advance Science and Technology, Research and Development Center, IRAN SSP. Now he is a dean of Intelligent Control and Robotics Lab. In addition to 7 textbooks, Farzin Piltan is the main author of more than 110 scientific papers in refereed journals. He is editorial review board member for 'international journal of control and automation (IJCA), Australia, ISSN: 2005-4297; 'International Journal of Intelligent System and Applications (IJISA)', Hong Kong, ISSN:2074-9058; 'IAES international journal of robotics and automation, Malaysia, ISSN:2089-4856; 'International Journal of Reconfigurable and Embedded Systems', Malaysia, ISSN:2089-4864. His current research interests are nonlinear control, artificial control system and applied to FPGA, robotics and artificial nonlinear control and IC engine modeling and control.

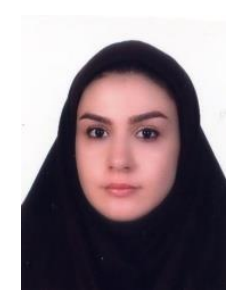

Mahsa Piltan is currently working as a co researcher in Control and Robotic Lab at the institute of advance science and technology, IRAN SSP research and development Center. She is a Master in field of communication engineering from Azad University, IRAN. Her current research interests are in the area of nonlinear control, artificial control system and robotics, and spherical motor.

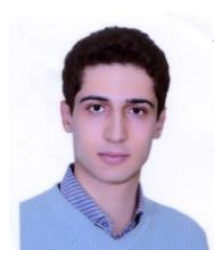

spherical motor.

Mojtaba Yaghoot is currently working as a primary researcher in Control and Robotic $\mathrm{Lab}$ at the institute of advance science and technology, IRAN SSP research and development Center. His current research interests are in the area of nonlinear control, artificial control system and robotics, and

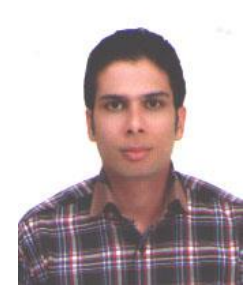

Meysam Esmaeili is currently working as a co researcher in Control and Robotic Lab at the institute of advance science and technology, IRAN SSP research and development Center. He is a Master in field of Electronic Engineering from Azad University, IRAN. His current research interests are in the area of nonlinear control, artificial control system and robotics, and spherical motor.

How to cite this paper: Mohammad Ali Tayebi, Farzin Piltan, Mahsa Piltan, Mojtaba Yaghoot, Meysam Esmaeili,"Design New Intelligent-Base Chattering Free Nonlinear Control of Spherical Motor", International Journal of Intelligent Systems and Applications(IJISA), vol.6, no.10, pp.55-66, 2014. DOI: 10.5815/ijisa.2014.10.08 